\title{
Recent Advances in Microwave Assisted Specimen Processing: Keeping it Cool.
}

\author{
Mark A. Sanders
}

Imaging Center, College of Biological Sciences, University of Minnesota, St. Paul, MN, 55108

Microwave energy as a method of rapid tissue processing is gaining increasing acceptance as an alternative to routine chemical processing in laboratories where rapid and accurate specimen processing is required. Conventional chemical fixatives and protocols used for standard preservation of biological specimens can result in alterations in morphology as a consequence of solubility and conformational changes of cellular constituents. These changes often result in morphological artifacts and compromised antigenicity of many tissue proteins. Conventional chemical fixatives in combination with temperature controlled microwave processing can distinctly shorten the processing time while maintaining better specimen preservation. In addition to tissue fixation, very low wattage in a dedicated laboratory microwave ( $<250$ watts, Pella 3450) can also be used to facilitate access of vital stains into developing embryonic organisms. The present study will have two objectives: to rapidly prepare specimens for fluorescent in situ hybridization and to utilize low wattage, temperature controlled microwave energy in in vivo experiments in plant and animal tissue. Low power microwave energy was used in conjunction with a recently developed microwave accessory called the ColdSpot ${ }^{\mathrm{TM}}$ (Ted Pella, Inc., Redding, CA) that creates a uniform environment of microwave irradiation to the samples.

This report demonstrates the use of low power microwave energy (250W or less) in conjunction with the ColdSpot $^{\mathrm{TM}}$ in an attempt to overcome the problems reported in the past (Boon and $\mathrm{Kok}^{1}$, Choi et al. ${ }^{2}$, Chicoine and $\mathrm{Webster}^{3}$ ). A number of different nucleic acid and protein probes were tested on known systems. Tissue culture cells, tissue sections and living organisms were labeled using the same basic protocol to examine the viability of a standardized approach. The reported results indicate low power microwave energy combined with better control of the microwave environment produce uniform results across a wide spectrum of ultrasturtural labeling techniques.

Additionally in order to attempt to improve co-localization studies of DNA and protein distributions, we are to developing a routine technique to co-localize specific nucleic acid sequences and multiple antigens simultaneously. We have applied this technique to a variety of specimens including nematode embryos (see Figure 1).

We have also found that under reduced wattage conditions ( 150 watts), living plant and animal tissue can be made "permeable" to fluorescent vital dyes such as the Syto series of nucleic acid stains (Molecular Probes, Eugene OR). Brief microwave treatment in the presence of the stain will greatly increase the depth and rate of penetration of the dye. Time-lapse fluorescence or confocal microscopy has shown promising results on several developing model systems including Arabidopsis trichomes, root tips, Drosophila and nematode embryos.

In conclusion, this method allows for the detection of multiple DNA sequences and antigens at the same tissue level with high sensitivity and good morphological preservation. Given the versatility of the system, it should be useful wherever simultaneous detection of multiple antigens and nuclear acid sequences is required. These procedures can have broad reaching applications in the preparation of specimens in clinical and research laboratory environments. 


\section{References:}

[1] Boon, M.E., and Kok, L.P., 1994. Microwaves for immunocytochemistry. Micron., 25:151-170.

[2] Choi, T.-S., Whittlesey, M.M., Slap, S.E., Anderson, V.M., Gu, J., 1995. Advances in temperature control of microwave immunohistochemistry. Cell Vision., 2:151-164.

[3] Chicoine, L., and Webster, P., 1998. Effect of microwave irradiation on antibody labeling efficiency when applied to ultrathin cryosections through fixed biological material. Microsc. Res. Tech., 42:24-32.
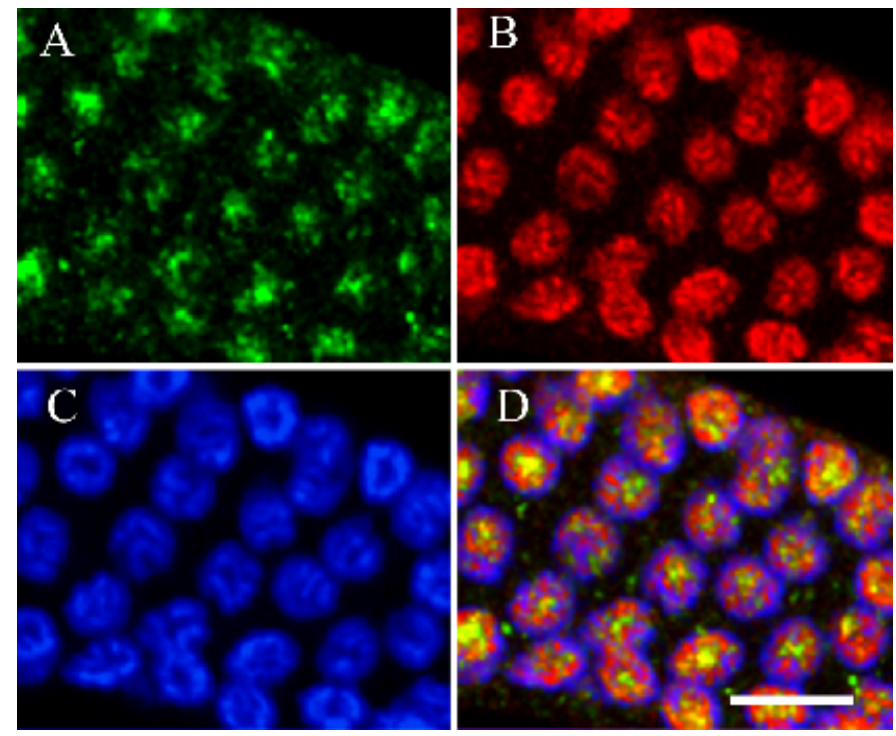

FIG. 1. LSCM image of c. elegans embryo triple labeled (D) with (A)Alexa 488 labeled D.v.TIM (RNAi), (B) Texas Red conjugate to anti-histone and (C) Syto 63 nucleic acid stain following microwave specimen processing utilizing a power level of $200 \mathrm{~W}$ and the ColdSpot ${ }^{\mathrm{TM}}$ (Ted Pella, Inc., Redding, CA). Scale bar $=20$ microns. 